\title{
Quantification of coil slippage during initial coiling with belt wrapper
}

\author{
Yonghui Park ${ }^{1, *}$ and Hyunchul Park ${ }^{2}$ \\ ${ }^{1}$ Pohang Institute of Metal Industry Advancement \\ 56, Jigok-ro, Namgu, Pohang, Korea \\ "Email: yonghuipark216@gmail.com \\ Phone: +82-54-279-9455, Fax : +82-54-279-9449 \\ ${ }^{2}$ Graduate School of Engineering \\ POSTECH, 77, Cheongam-ro, Namgu, Pohang, Korea
}

\begin{abstract}
The finite element model and mathematical model for the coiling process with the belt wrapper have been developed by simplifying the belt wrapper to copy the strip head end positioning on free tension reel shortly, known as the initial coiling. The models have tried to quantify the coil slippage that makes a scratch on the coil. However, it is hard to compare the models to the real operation due to some assumptions. Moreover, computations is increased dramatically if the model includes the real operation such as non-linear property of the belt wrapper. This study suggests new modelling approach without the belt wrapper to quantify the coil slippage according the compression on the coil. Instead of the belt wrapper, all nodes of the strip are controlled by specific $x-y$ displacements as the belt wrapper compresses the strip coil. Different $x-y$ displacements of each node can change the magnitude of the compression by the belt wrapper. A finite element model of the initial coiling was simulated by the modelling approach and Model Change technique in ABAQUS, and discontinuous processes including removal of the belt wrapper and the application of coiling tension, $\sigma$ are proceeded in consecutive order. By doing parametric analysis regarding the radial deformation of the coil by the belt wrapper, $r_{d}$ and $\sigma$, the coil slippage happens frequently when $\sigma$ is below critical value or when $r_{d}$ is large. This phenomenon is similar to the spring back, so $\sigma$ should be high or should be applied before the belt wrapper is removed. This indicates that, $r_{d}$ should be manipulated carefully because the coil slippage happens as $r_{d}$ increases. Besides the initial coiling, the modelling approach can be applied to other problems that need many computations such as the 300 layers of strip lamination.
\end{abstract}

Keywords: Belt wrapper; Initial coiling; Spiral coil; Coil slippage; Finite element method.

\section{INTRODUCTION}

Finding ways to fix the head end of a strip on a sleeve or mandrel is an important factor to determine coiling condition in the coiling process. To prevent the coiling process from physical problems such as coil slippage, the strip is clamped on the mandrel or sleeve, or fastened with a piece of adhesive tape; then, the sleeve rotates to accumulate coil layers [1]. Due to its simple mechanism, most coiling research have focused on its optimisation. For instance, the analytical model and finite element model to calculate stress distribution of the mandrel, sleeve, and coil [2,3], optimisation of flatness of strip during the coiling process based on evolutionary algorithms regarding stress distribution in the strip [4], 
stress distribution of the coil with different temperature distribution [3], and stress distribution due to the flatness and crown of the coil [5] have been studied. In contrast, the coiling process with belt wrapper has been developed based on the engineer's technical experience. From several patents regarding new mechanisms of the belt wrapper $[6,7]$, experimental approaches have been conducted to find out the forcing relationship between the belt wrapper and the strip and to express the relationship as a mathematical expression to be used well $[8,9]$. These approaches are good for only manipulating their facilities when they always manufacture same coil. However, it takes a lot of money and time to investigate a physical phenomenon when they want to change the operating condition or design variable. For instance, the coil slippage makes scratches on the coil when coiling tension $\sigma$ is not sufficient, then the coil is not be provided to customers. A thin strip is hard to control the coil slippage compared to a thick strip that has a thickness over $3 \mathrm{~mm}$. This limitation can be solved with a computational approach such as the finite element method [10-12]. Recently, our research group has presented the finite element model and the analytical model to calculate stress distribution during the initial coiling and to find out whether the coil comes untied or not $[13,14]$. By using these models, a parametric study regarding the magnitude of the compression by the belt wrapper can be carried out easily. However, the models do not have the non-linear material property of the rubber and must be improved by copying real operating conditions. But, the finite element model take a long time to calculate the stress and other physical quantities as the non-linear behaviours are included.

To simplify the non-linear behaviours of the belt wrapper, this paper suggests new modelling approach for quantifying the compression on the coil by controlling the displacement of all nodes on the centre of the strip instead of the belt wrapper. This method can reduce many computations for contact relationship among coil layers, so the finite element model can determine whether the magnitude of the compression is proper to the coil slippage or not quickly. To control the displacement of the strip efficiently, the MATLAB code and ABAQUS input file were introduced on the calculation of $x$ and $y$ coordinates of all nodes and the definition of dynamic analysis in the ABAQUS automatically. To minimise the coil slippage that makes the scratch, parametric study was conducted about the condition of the coil slippage with different $\sigma$ and the radial deformation of the coil by the belt wrapper $r_{d}$.

\section{METHODS AND MATERIALS}

\section{System Modelling and Code}

In the steel manufacturing industry, the initial coiling is called as strip head end positioning on free tension reel, but we used the initial coiling shortly. The initial coiling is operated with the belt wrapper, sleeve, and strip (Fig. 1) [13]. As the belt wrapper compresses the outside of the strip and rotates with the sleeve at the same direction, the strip is coiled about 2 3 turns between the sleeve and belt wrapper. Because the first layer is stacked on the outer surface of the sleeve directly, the coil has a spiral shape, not the circle. Also, each contact layer has the pressure along the radial direction and frictional force along the tangential direction. After 2 3 turns of the coil, $\sigma$ is applied to the opposite end of the strip and the belt wrapper is removed from the coil to wind the strip rapidly. In this mechanism, the coil slippage takes place discontinuously according to coiling conditions, because the left end of the strip is not fixed on the outside of the sleeve. The summation of the frictional forces resists the coil slippage. Based on this difficulty and high computations for the behaviour of the belt wrapper, we skipped the deformation of 
the coil with the belt wrapper during the initial coiling. Then, to find out the condition of the coil slippage, we have developed a new ABAQUS input file that coils the strip by applying pre-defined coordinates of each node on the centre line of the strip without any contact definition during the initial coiling. The pre-defined coordinates can be changed according to coiling conditions such as the number of coil layer and $r_{d}$.

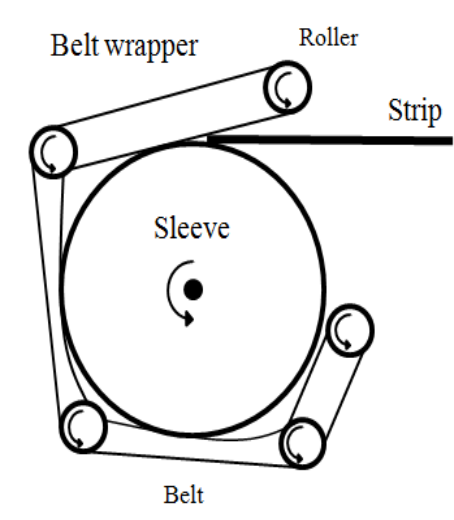

(a) Winding step

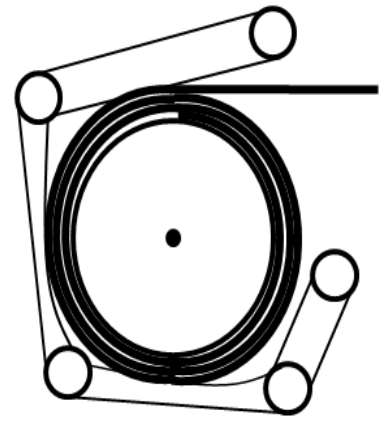

(b) Free and tension on step

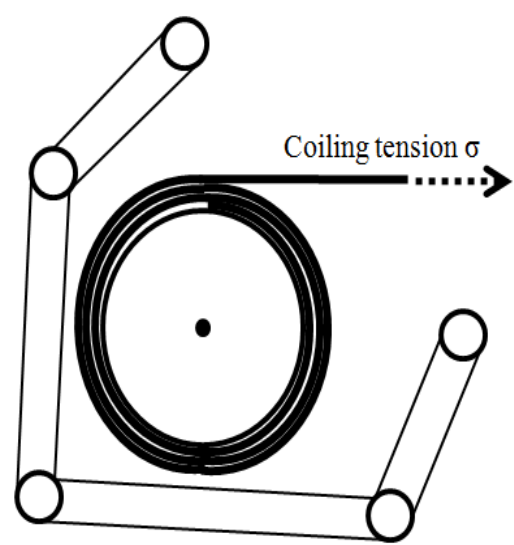

(c) Coiling step

Figure 1. Diagram of the initial coiling; (a) The strip is wound between the belt wrapper and sleeve by compression and frictional forces from the belt wrapper; (b) The belt wrapper and the sleeve do not rotate after $2 \sim 3$ turns of the coil; (c) $\sigma$ is applied at the opposite end of the strip, and the belt wrapper is removed from the coil because the coil has sufficient frictional force to prevent itself from the slippage.

\section{Finite element model}

The finite element model has the sleeve and strip in the 2-dimensional space. The sleeve is rigid with the wire shape. The strip has a uniform mesh along the lateral and longitudinal directions (Fig. 2). The number $N_{\text {long }}$ of the mesh along the longitudinal direction determines the number of the winding steps. For instance, the analysis model have 11 winding steps during the initial winding if the $N_{\text {long }}$ is 11 .

\section{Winding step}

In the winding step, the strip is coiled along the sleeve gradually. Each winding step moves the $i$ th centre node of the strip along the longitudinal direction respectively. For instance, the left end of the node is located on the specific coordinates when the winding first step, and the $i$ th of the node from the left end of the node is located on the specific coordinates during the winding $i$ th step. $i$ is an integer from 1 to $N_{\text {long. Through the }}$ winding steps, some nodes are coiled and the other nodes are left as a straight line (Fig. 2). To apply the coordinates of each node respectively, the boundary conditions about the $x$ and $y$ displacements are defined in each winding step. In these steps, general contact between the sleeve and strip is not defined. In other words, penetrations among contact layers are shown visually, but they are not effective to each other to create the pressure or frictional force. This method is similar to the initial inference fit problem among two rings $[15,16]$. According to $r_{d}$, the shape of coil and the initial penetration between each strip coil are different. 
(a) At the initial state

(b) At the end of the winding step

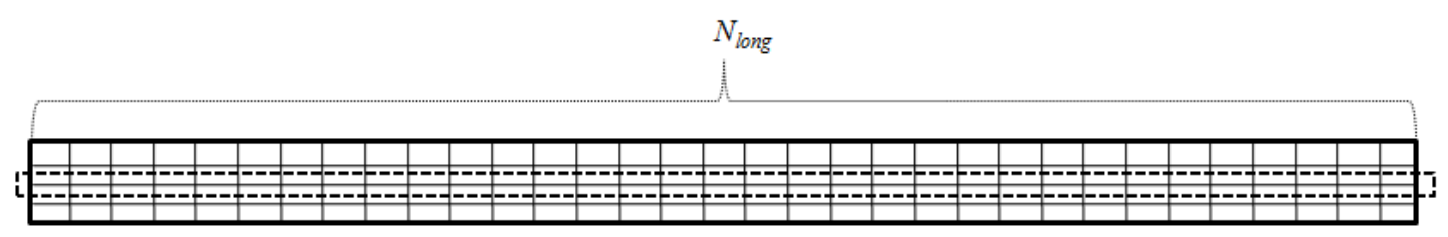

(c) The mesh grid of the strip

Figure 2. The finite element model of the initial coiling; (a) The initial location of the sleeve and the strip in the model; (b) At the end of the winding step, the strip is wound around the sleeve; (c) The mesh grid of the strip about the longitudinal direction and the strip thickness direction, and the centre nodes of the strip that are placed at specific coordinates by the ABAQUS code.

\section{Free and tension on step}

In this step, $\sigma$ is applied to the right end of the strip and the definition of the general contact is applied to calculate the pressure and frictional forces among contact layers from the winding step. Because the boundary conditions for all nodes of $N_{\text {long }}$ are still effective in the free and tension on step, $\sigma$ cannot draw the strip. The reason that $\sigma$ is applied in this step is to prevent the simulation from divergence. If $\sigma$ is applied in next step, convergence problem can be serious with the fluctuation of the strip caused by removal of the boundary conditions for all nodes of $N_{\text {long. }}$. In relation tothe general contact, hard contact for the normal direction and penalty method for the tangential direction are used. Except for the nodes of $N_{\text {long, }}$, all nodes of the strip are calculated in equilibrium states by the interference fit in this step. For instance, the strip coil is deformed along the radial direction to fit boundaries among different contact layers.

\section{Coiling step}

The boundary conditions on the nodes of $N_{\text {long }}$ are not effective in this step. Finally, the model has the only boundary condition on the center of the sleeve. The pressure among contact layers in the strip coil try to become united to make the initial state of the strip, and $\sigma$ tries to prevent the strip coil from the coil slippage. According to $r_{d}$ and $\sigma$, the coil slippage is varied, so a parametric study should be conducted to identify the relationship.

\section{Code to Develop Analysis Model}

To conduct the parametric study efficiently, the generation of the ABAQUS input file can be a good method to change design variable or other parameters [17]. For instance, the same command lines of the ABAQUS input are used in our problem, so we need to modify a few command lines for the parametric analysis. The ABAQUS input file has part with node \& element, assembly, set, material, interaction, and step commands sequentially. In our model, the node label and boundary condition should be modified for the parametric study. When the mesh of the strip is changed, the node labels of $N_{\text {long }}$ and 
these boundary conditions changed at the same time. So, the MATLAB code that tracks the node label and writes an ABAQUS input file automatically, was suggested.

\section{Definition of the coordinates of the strip node of $N_{l o n g}$}

First, ABAQUS CAE file was established with the part and mesh, and was exported into the input file. In the model, the strip nodes of $N_{\text {long }}$ have specific node labels and their initial positions. By using the node and position information, each node's $x$ and $y$ displacements are derived to coil the strip as the spiral shape [18, 19]. Eqs. (1-7) were defined to draw the strip coil that is compressed. About input variables in Eqs. (1-7), the longitudinal length of the strip $L_{l o n g}$, the number of the coil layer $C N$, the thickness of the strip $t$, the outer radius of the sleeve $r_{o \text {,sleeve }}, r_{d}$, and $N_{\text {long }}$ were defined. $r_{d}$ affects the radius of the coil and pitch $p$ (Fig. 3).

$$
\begin{gathered}
r_{i, \text { belt }, \text { ini }}=r_{o, \text { sleeve }}+t \times C N \\
r_{i, \text { belt }}=r_{i, \text { belt }, \text { ini }}-r_{d} \\
t_{\text {deform }}=\left(r_{i, \text { belt }}-r_{o, \text { sleve }}\right) / C N \\
r_{c, \text { coil } 1}=r_{o, \text { sleeve }}+t_{\text {deform }} / 2 \\
r_{c, \text { coilCN }}=r_{i, \text { belt }}-t_{\text {deform }} / 2 \\
p=t-\left(r_{d} / C N\right) \\
r_{\text {coil }}(\theta)=r_{c, \text { coil } 1}+\frac{p}{2 \pi} \theta
\end{gathered}
$$

where $r_{i, \text { belt,ini }}$ is the inner radius of the belt wrapper without $r_{d}$ and is the same as the outer radius of the coil $r_{o, \text { coil }}, r_{i, \text { belt }}$, is the inner radius of the belt wrapper with $r_{d}, t_{\text {deform }}$ is deformed thickness of each coil layer, $r_{c, \text { coill }}$ is the centre radius of the first coil, and the $r_{c, \text { coilcN }}$ is the centre radius of the $C N$ th coil, $p$ is the pitch of the spiral coil, $r_{\text {coil }}$ the centre radius of the coil according to $\theta$. After determining the design variable and coiling status, each node should be placed at a specific location.

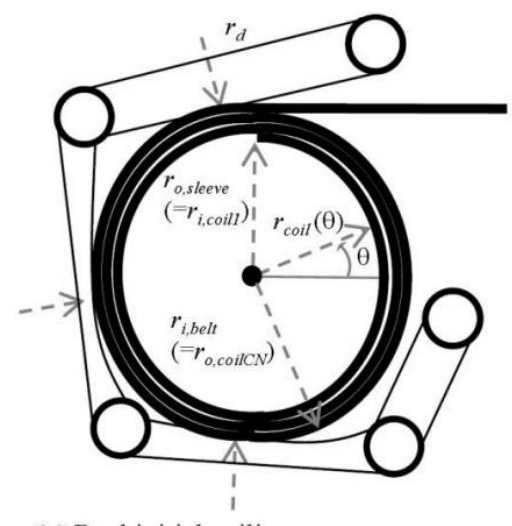

(a) Real initial coiling process

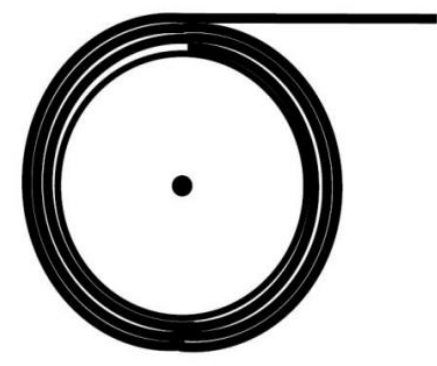

(b) Virtual initial coiling process

Figure 3. The spiral shape of the coil; (a) The initial coiling with the belt wrapper in real operation; (b) The initial coiling by specifying the coordinates of the strip nodes without the belt wrapper

To locate them, node displacement calculation code was established by Eqs. (813). Eqs. (8-13) are repeated at $N_{\text {long }}$ to calculate different $x$ and $y$ displacements for 
different node of $N_{\text {long }}$ (Fig. 4). Llong nodes have $2 N_{\text {long }}$ displacements for the boundary condition.

$$
\begin{array}{r}
L_{\text {coil }}=\int_{0}^{2 \pi \times C N}\left(\left(r_{\text {coil }}(\theta)\right)^{2}+\left(\frac{d r_{\text {coil }}(\theta)}{d \theta}\right)^{2}\right) d \theta \\
L_{\text {refer }}=\sqrt{(x(i)-x(1))^{2}+(y(i)-y(1))^{2}}
\end{array}
$$

if $L_{\text {refer }}<=L_{\text {coil }}$, solve the equation to find out $\theta_{\text {refer }}$

$$
\begin{gathered}
\int_{0}^{2 \pi \times \theta_{\text {refer }}}\left(\left(r_{\text {coil }}(\theta)\right)^{2}+\left(\frac{d r_{\text {coil }}(\theta)}{d \theta}\right)^{2}\right) d \theta-L_{\text {refer }}=0 \\
x_{t}(i)=-r_{\text {coil }}\left(\theta_{\text {refer }}\right) \times \cos \left(\theta_{\text {refer }}\right) \\
y_{t}(i)=r_{\text {coil }}\left(\theta_{\text {refer }}\right) \times \sin \left(\theta_{\text {refer }}\right)
\end{gathered}
$$

if $L_{\text {refer }}>L_{\text {coil }}$

$$
\begin{gathered}
L_{\text {rest }}=L_{\text {refer }}-L_{\text {coil }} \\
x_{t}(i)=-r_{\text {coil }}(2 \pi \times C N) \times \cos (2 \pi \times C N)+L_{\text {rest }} \\
y_{t}(i)=r_{\text {coil }}(2 \pi \times C N) \times \sin (2 \pi \times C N)
\end{gathered}
$$

where $L_{\text {coil }}$ is the length of the coil, $L_{\text {refer }}$ is the distance between the left end node of the strip and the $i$ th node of the strip, $x_{t}$ and $y_{t}$ are displacements of specific node for the spiral shape of the coil, $L_{\text {rest }}$ is the distance between the end node of the strip and the end node of the coil.

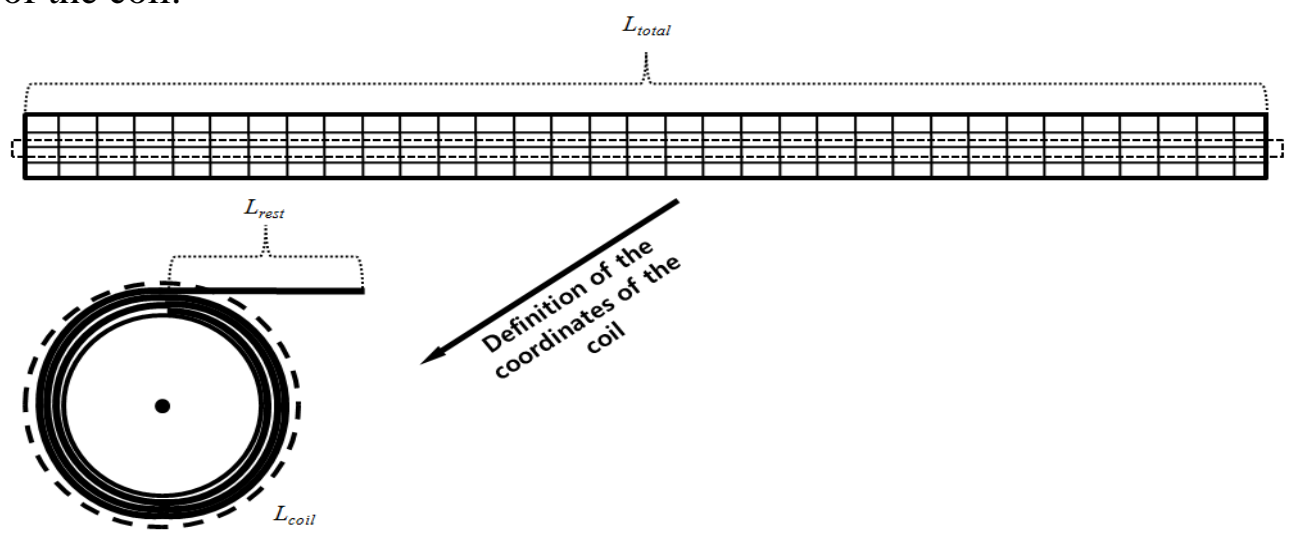

Figure 4. $L_{\text {coil }}$ and $L_{\text {rest }}$ at the end of the winding step.

\section{Definition of the node sets}

The second code defines the node number of the nodes of $N_{\text {long }}$ in the ABAQUS input file, because the node selection in the ABAQUS CAE is not efficient. For instance, we have to define 1000 node sets to define the $x$ and $y$ displacements of the boundary conditions when $N_{\text {long }}$ is 1000 . Fortunately, the ABAQUS specifies the node number by following a specific rule. In the simulation, the nodes of numbers were $(2,305,304,303$, $\ldots . ., 9,8,7,1)$ from the left end of the strip node. Except the both end of the strip, the number is decreased as -1 from the left to the right. For the step command lines for the 
ABAQUS input file, the MATLAB code 'nset_code_new' can be derived in APPENDIX $[20,21]$.

\section{Definition of the winding steps}

The reason that the model has $N_{\text {long }}$ winding steps is to prevent the coil from twisting. When all $x$ and $y$ displacements of the boundary condition are applied at the same time, the coil twisted and the analysis diverged. Before the free and tension on step and coiling step in the ABAQUS input file, all winding steps have the same command lines, except the value of the $x$ and $y$ displacements. In the first winding step, the definition of the general contact is removed to establish the initial penetration problem corresponding the $r_{d}$. The general contact applied in the free and tension on step. The MATALB code 'winding_abaqus_inp_code' is in APPENDIX to define the winding steps. In this code, the target $x$ and $y$ displacements from the Section 2.3.1 are needed. All command lines are located between the initial interaction command lines and the free and tension on the step command lines.

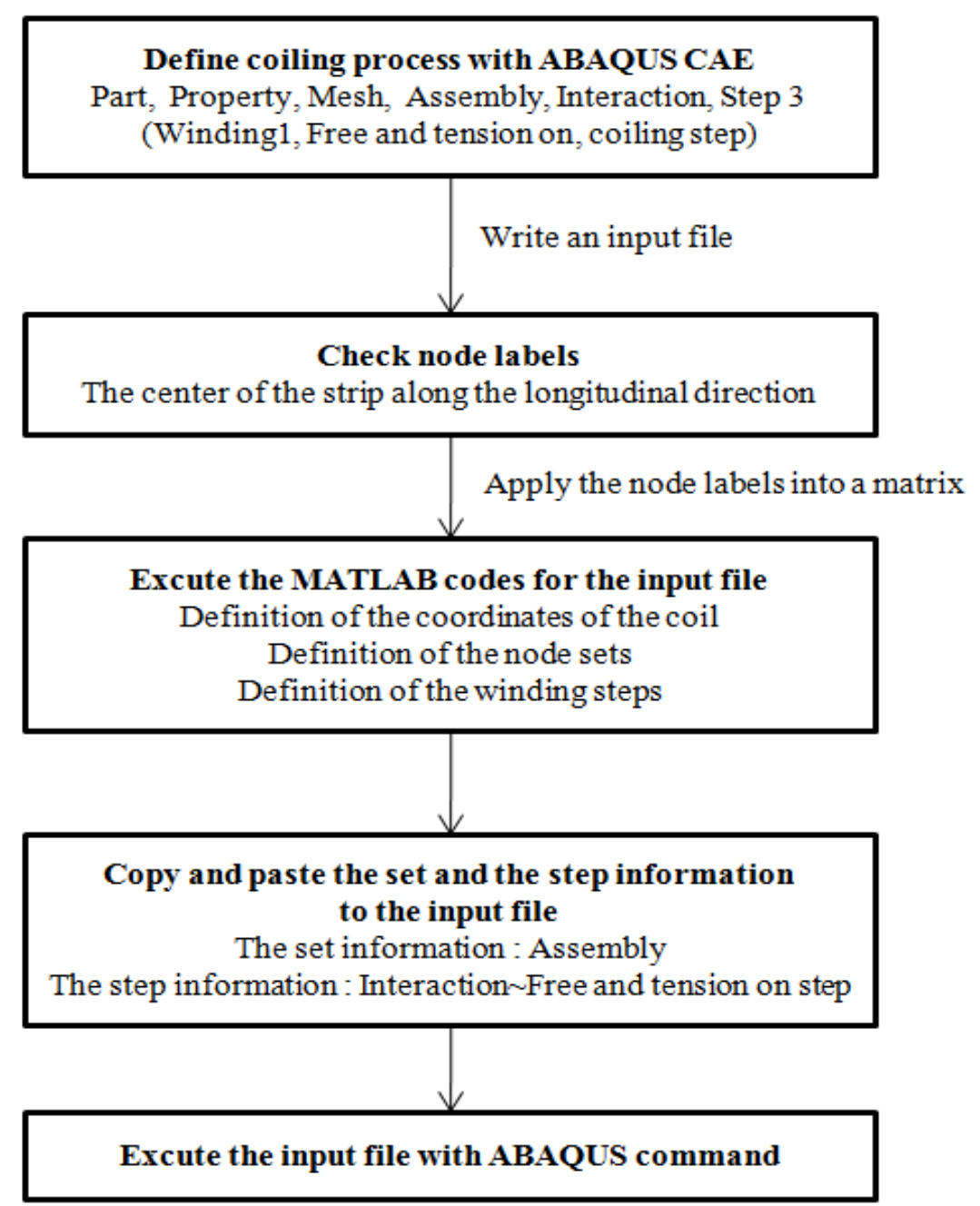

Figure 5. Flow chart of the command.

\section{Simulation Process}

Figure 5 is the flow chart of the command between the initial coiling model and MATLAB codes. Even though some MATLAB codes and ABAQUS input files need to 
be changed, parametric study can be conducted according to $r_{d}, r_{o, \text { sleeve, }}$, and material properties. This work can handle engineering problems such as defining operating condition of the initial coiling rapidly.

Table 1. Design variables and operating conditions of the simulation.

\begin{tabular}{lc}
\hline & Value \\
\hline Density of the strip, $\rho_{\text {strip }}$ & $7890 \mathrm{~kg} / \mathrm{m}^{3}$ \\
The modulus of elasticity of the strip, $E_{\text {strip }}$ & $214 \times 10 \mathrm{GPa}$ \\
Poisson's ratio of the strip, $v_{\text {strip }}$ & 0.28 \\
The length of the strip, $L_{\text {total }}$ & $10 \mathrm{~m}$ \\
The thickness of the strip, $t$ & $1 \mathrm{~mm}$ \\
The outer radius of the sleeve, $\mathrm{r}_{\mathrm{o}, \text { sleeve }}$ & $0.324 \mathrm{~m}$ \\
Tangential behaviour for the contact property & Penalty (The coefficient of \\
Normal behaviour for the contact property & friction, $\mu=0.1$ ) \\
The number of nodes of the strip about the & 301 \\
longitudinal direction, $N_{\text {long }}$ & \\
The number of nodes of the strip about the strip & 5 \\
thickness direction, $N_{\text {short }}$ & 3 \\
The number of coil layer, $C N$ & ENCASTRE \\
Boundary condition on the center of the sleeve &
\end{tabular}

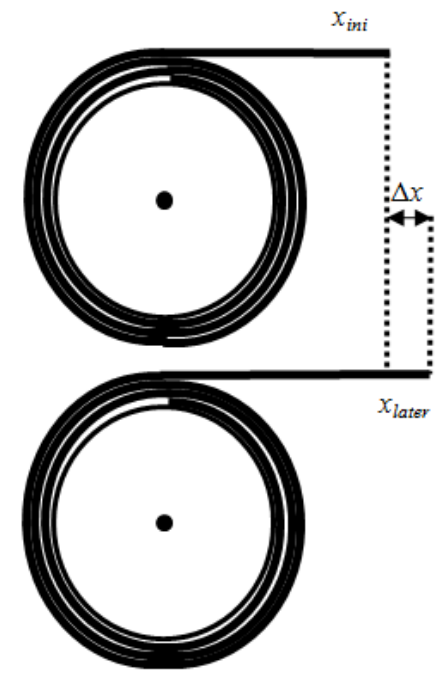

Figure 6 . The difference $\Delta x$ between the initial $x$ coordinate $x_{i n i}$ and the final $x$ coordinate $x_{\text {later }}$ of the right end of the strip.

\section{Simulation Condition}

Table 1 is the list of design variables and operating conditions to simulate the initial coiling model [13]. To find out the system performance and sensitivity according to parameter, the parameter should be varied with different MATLAB code and ABAQUS input file. In this paper, the coil slippage was investigated depending on $r_{d}$ and $\sigma$. The coil slippage is necessary to find out the coil is fixed on the sleeve during the coiling step. $r_{d}$ was applied into the codes as $0 \mathrm{~m}, 0.000001 \mathrm{~m}, 0.000003 \mathrm{~m}, 0.00001 \mathrm{~m}, 0.00003 \mathrm{~m}$, and $0.00006 \mathrm{~m}$. Apart from that, $\sigma$ is varied from $0 \mathrm{MPa}$ to $50 \mathrm{MPa}$. The $x$ displacements of 
the right end of the $N_{\text {long }}$ th strip node $x_{i n i}, x_{\text {later }}$, and $\Delta x$ were checked to know whether the coil is slip or not during the parametric study (Figure 6).

\section{RESULTS AND DISCUSSION}

\section{The Coil Slippage according to $\sigma$}

$\sigma$ is an important factor to determine the coil slippage. When the coil slippage happened during the coiling step, $r_{d}$ was too large in the winding steps or $\sigma$ was too low to grab the fluctuation of the coil between the free and tension on step and coiling step. When the boundary conditions of the strip nodes are removed at the end of the free and tension on step, the strip coil has spring back effect that raises the expansion of the strip coil. The spring back effect is extreme when $\sigma$ is not applied to the strip before the belt wrapper is removed [13]. If $\sigma$ fails to stop the spring back effect, the coil slip and come untied (Figure 7).

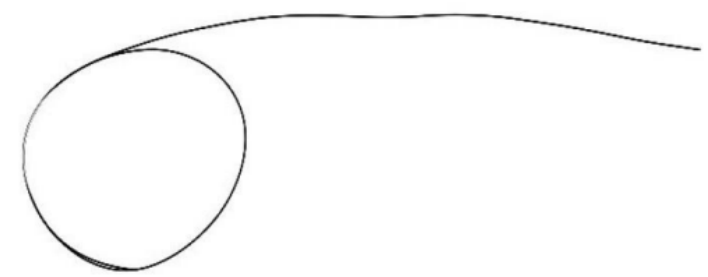

(a) The coil slippage

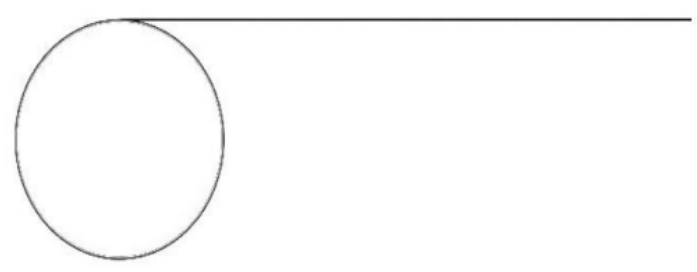

(b) The non coil slippage

Figure 7. Deformation of the strip according to $\sigma$ during the coiling step; (a) The strip comes untied because $\sigma$ is not sufficient to stop the spring back effect; (b) The strip has the spiral shape even though the belt wrapper is removed.

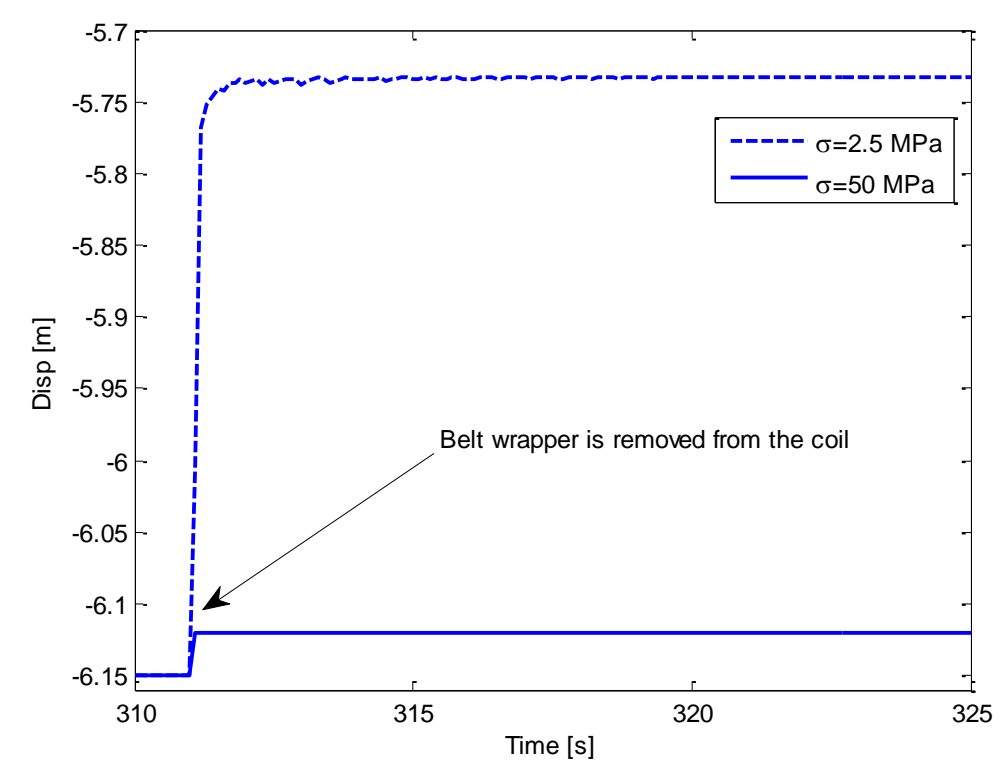

Figure 8. $x$ displacement of the right end of the $N_{\text {long }}$ th strip node during the coiling step.

Figure. 8 shows the $x$ displacements of the right end of the $N_{\text {long }}$ th strip node when $\sigma$ is $50 \mathrm{MPa}$ or $2.5 \mathrm{MPa}$, and $r_{d}$ is $0 \mathrm{~m}$. When $\sigma$ is lower than $2.5 \mathrm{MPa}$, the coil slippage was extreme as shown in Figure 7 (a). When $\sigma$ is $2.5 \mathrm{MPa}$, the $x$ displacement is converged with a large variation. In contrast, the coiling step can coil the strip after the free and 
tension on step with small variation when the $\sigma$ is higher than $2.5 \mathrm{MPa}$. In other words, when the critical value $\sigma_{\text {cri }}$ of $\sigma$ is calculated without the coil slippage, any $\sigma$ that is higher than $\sigma_{\text {cri }}$ not raise the coil slippage after the initial coil slippage.

\section{The variation of the coil slippage according to $\sigma$ under the non-coil slippage}

To investigate how the coil slippage is stopped according to $\sigma$, the $x$ displacement of the right end of the $N_{\text {long }}$ th strip node $\Delta x$ were acquired when $\sigma$ is higher than $\sigma_{\text {cri. }} \Delta x$ is $\left(x_{\text {later }}\right.$ - $\left.x_{\text {ini }}\right) . \Delta x$ is converged at the different value according to $\sigma$. If the coil slippage does not appear, $\Delta x$ is not be changed with constant $0 \mathrm{~m}$. Even though $\sigma$ is extremely high, the coil slippage only happens temporarily. As $\sigma$ increases, $\Delta x$ converges at the small value rapidly. Fig. 9 is $\Delta x$ distribution according to different $\sigma$ when $r_{d}$ is $0 \mathrm{~m}$. $\sigma$ was varied from $0 \mathrm{MPa}$ to $50 \mathrm{MPa}$. As $\sigma$ increases, $\Delta x$ goes down. The difference can be used to quantify the surface quality on the coil in the initial coiling to minimise the coil slippage with the low electrical energy.

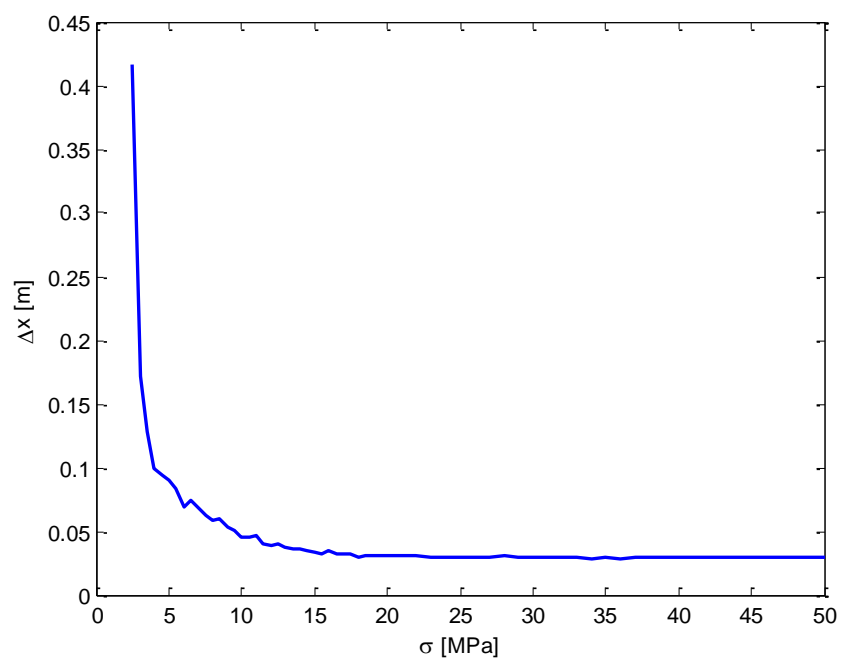

Figure 9. The difference of $\Delta x$ between $x_{i n i}$ and $x_{\text {later }}$ of the right end of the $N_{\text {long }}$ th strip node according to $\sigma$.

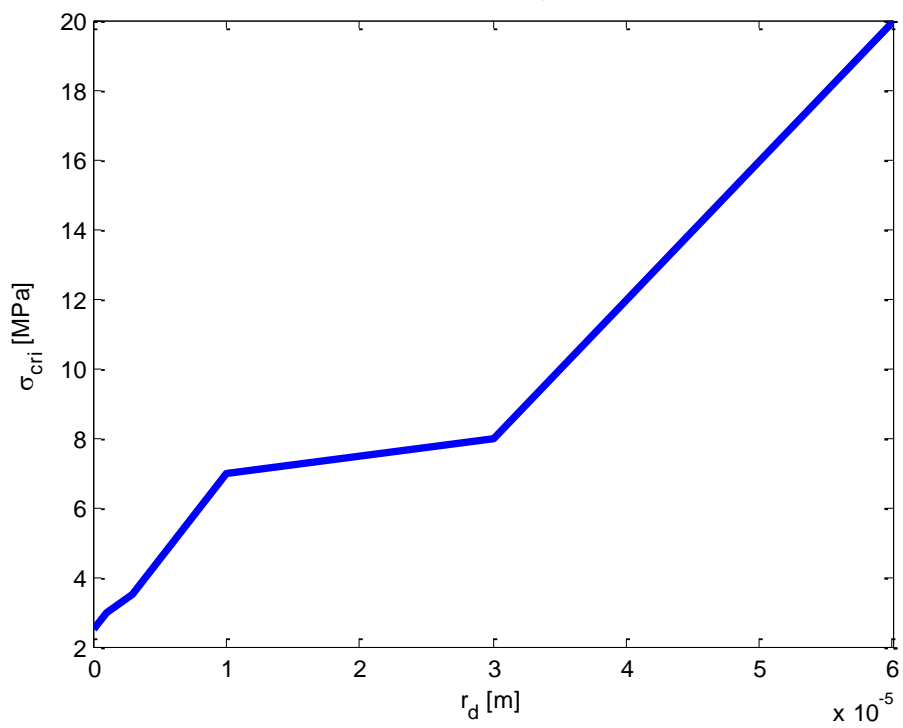

Figure 10. $\sigma_{\text {cri }}$ distribution according to $r_{d}$. 


\section{The relationship between $r_{d}$ and $\sigma$}

In this section, $\sigma_{\text {cri }}$ and $\Delta x$ were investigated by changing $r_{d}$ and $\sigma$. Fig. 10 is $\sigma_{\text {cri }}$ distribution according to $r_{d}$. As $r_{d}$ increases, the spring back effect also increases, so the system needs high $\sigma$ to avoid the coil from the slippage. The other method is applying $\sigma$ to the strip before the belt wrapper is removed [13]. To quantify the initial coiling, the system can measure small $r_{d}$, because the strip is steel and $\sigma_{\text {cri }}$ changes from $2.5 \mathrm{MPa}$ to $20 \mathrm{MPa}$ when $r_{d}$ changes from $0 \mathrm{~m}$ to $0.00006 \mathrm{~m}$. Fig. 11 is $\Delta x$ according to different $r_{d}$ and same $\sigma$. As $r_{d}$ increases, $\Delta x$ also increase.

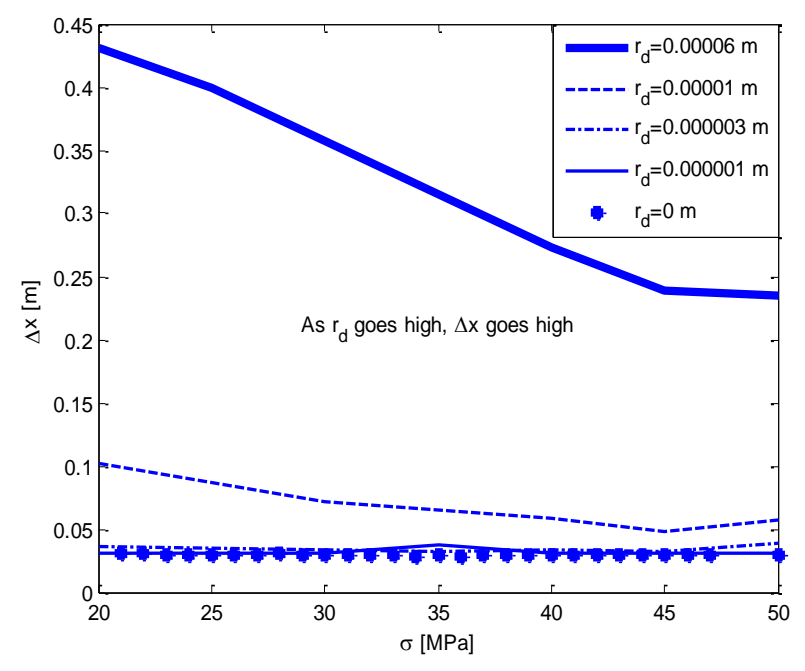

Figure 11. The difference $\Delta x$ according to $r_{d}$.

\section{CONCLUSIONS}

This study suggests the ABAQUS model and MATLAB code to figure out operating condition of the coil slippage. In order to reduce many computations due to the non-linear property of the initial coiling, the spiral shape of the coil was formulised and different coiling conditions such as the coiling layer $N_{\text {coillayer }}$ and the radial deformation of the coil by the belt wrapper $r_{d}$ were applied to the model by a parametric study to calculate $\sigma_{\text {cri }}$. As $r_{d}$ increased, the spring back effect raised the coil slippage. However, the coil slippage was stopped due to increase in the frictional force when $\sigma$ is larger than $\sigma_{\text {cri, }}$, even though extremely high $\sigma$ is applied. For instance, $r_{d}$ should be manipulated carefully, because the coil slippage happens as $r_{d}$ increases. From the findings, the initial coiling can be designed and operated by the parametric study about other coiling parameters with the suggested ABAQUS model and MATLAB code. Not only the initial coiling, the modelling approach can also be applied to other problems that need many computations such as the 300 layers of strip lamination with the belt wrapper. In the future, the surface quality on the coil will be examined with the model to find out the minimum $\sigma$ in the initial coiling.

\section{ACKNOWLEDGEMENTS}

The authors thanks to Pohang Institute of Metal Industry Advancement, South Korea and Graduate School of Engineering, POSTECH, Pohang, South Korea for their financial and laboratory facilities.

\section{REFERENCES}


[1] Roberts WL. Hot rolling of steel: CRC Press; 1983.

[2] Wang Y-Q, Li L, Yan XC, Luo YX, Liang WU. Modeling of stress distribution during strip coiling process. Journal of Iron and Steel Research, International. 2012;19:6-11.

[3] Park YH, Park K, Won SY, Hong WK, Park HC. Stress analysis model of strip winding system with a sleeve for a coil of thin stainless steel. Journal of Iron and Steel Research, International. 2017;24:1-7.

[4] Pholdee N, Bureerat S, Park WW, Kim DK, Im YT, Kwon HC, et al. Optimization of flatness of strip during coiling process based on evolutionary algorithms. International Journal of Precision Engineering and Manufacturing. 2015;16:14939.

[5] Park WW, Kim DK, Im YT, Kwon HC, Chun MS. Effects of processing parameters on elastic deformation of the coil during the thin-strip coiling process. Metals and Materials International. 2014;20:719-26.

[6] J PA. Belt wrappers. Google Patents; 1967.

[7] Herman K, Gerhard S. Belt wrapper for a coiler for the winding of rolled steel strip. Google Patents; 1969.

[8] Xi C, Juan LY, Lei Y. Design and calculation of belt wrapper2011.

[9] $\mathrm{Su} \mathrm{Y,} \mathrm{Tao} \mathrm{CM,} \mathrm{Mao} \mathrm{C,} \mathrm{Xiu} \mathrm{DB.} \mathrm{Analysis} \mathrm{of} \mathrm{force} \mathrm{distribution} \mathrm{on} \mathrm{belt} \mathrm{wraper}$ ofcoiler of cold rolling mill and discussion on its optimization. Wuhan Iron and Steel Corporation Technology. 2012.

[10] Rashid H, Abdullah AH, Noh MHM, Hamid AHA, Abidin NMZ. Design of a superbike paddock stand using cad and cae tools. Proceedings of The World Engineering Congress 2010, Conference on Aerospace and Mechanical Engineering2010. p. 267-74.

[11] Wor LC, Rahman MM. Stress behavior of tailor-welded blanks for dissimilar metals using finite element method. International Journal of Automotive and Mechanical Engineering. 2015;11:2541-54.

[12] Kadirgama K, Noor MM, Rahman MM, Harun WSW, Haron CHC. Finite element analysis and statistical method to determine temperature distribution on cutting tool in end-milling. European Journal of Scientific Research. 2009;30:451-63.

[13] Park Y, Park H. Development of computational models for coiling process with the belt wrapper. Metallurgical and Materials Transactions B. 2016;47:2699-704.

[14] Rahman MM, Kadirgama K, Noor MM, Rejab MRM, Kesulai SA. Fatigue life prediction of lower suspension arm using strain-life approach. European Journal of Scientific Research. 2009;30:437-50.

[15] Jain LK, Mai YW. Stresses and deformations induced during manufacturing. Part i: Theoretical analysis of composite cylinders and shells. Journal of Composite Materials. 1997;31:672-95.

[16] Hibbitt, Karlsson, Sorensen. Abaqus: Example problems manual: Hibbitt, Karlsson \& Sorensen; 2001.

[17] Hibbit KS. Abaqus scripting user's manual (version 6.3). Pawtucket: HKS. 2002.

[18] Du R, Xie L. The mechanics of the spiral spring. The mechanics of mechanical watches and clocks: Springer; 2013. p. 89-113.

[19] Hammelmüller F, Holl HJ. The effect of variable eccentricity onto the oscillations in a coiling process. PAMM. 2003;3:104-5. 
[20] Djojodihardjo H. Influence of the earth's dominant oblateness parameter on the low formation orbits of micro-satellites. International Journal of Automotive and Mechanical Engineering. 2014;9:1802.

[21] Etter DM, Kuncicky DC, Hull DW. Introduction to matlab 6: Pearson Education; 2004.

\section{APPENDIX}

\section{nset_code_new}

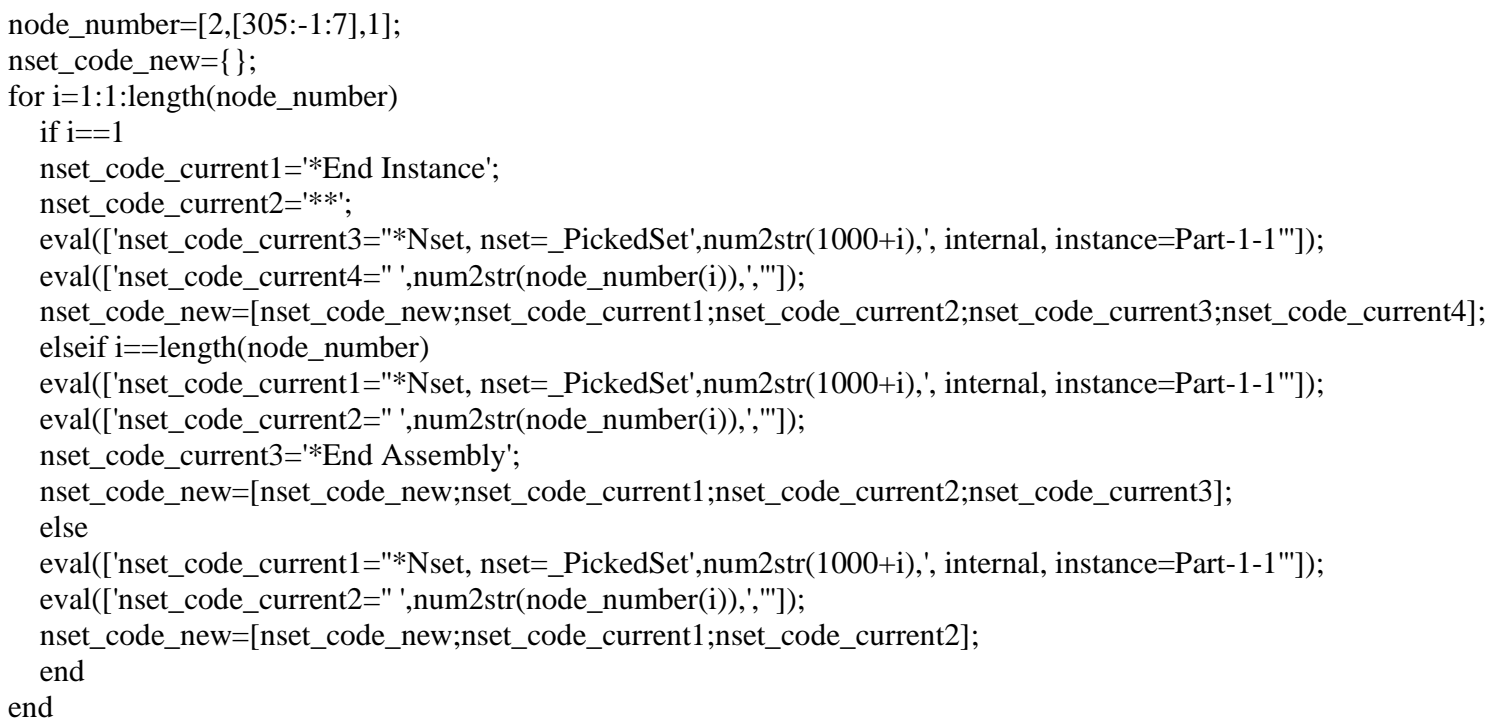

\section{winding_abaqus_inp_code}

load('RESULT_COORDINATE.mat')

node_number $=[2,[305:-1: 7], 1]$;

increment $=1000000$;

step_ini $=0.1$;

time_end $=1$;

step_min=1e-05;

output_interval $=5$

step_code_new $=\{\}$;

for $\mathrm{i}=1: 1$ :length(node_number)

if $i==1$

eval(['step_code_current1="*Step, name=Winding',num2str(i),', nlgeom=YES, inc=',num2str(increment),"'])

step_code_current $2=$ '*Dynamic';

eval(['step_code_current3='",num2str(step_ini),,','num2str(time_end),,','num2str(step_min),"''])

step_code_current $4={ }^{*} * *$ BOUNDARY CONDITIONS';

eval(['step_code_current5="** Name: BC-',num2str(i),' Type: Displacement/Rotation"']);

step_code_current $6=$ '*Boundary';

eval(['step_code_current7="_PickedSet',num2str(1000+i),', 1, 1, ',num2str(RESULT(i,1),10),"'"])

eval(['step_code_current8="_PickedSet',num2str(1000+i),', 2, 2, ',num2str(RESULT(i,2),10),"']])

step_code_current $9=' *$ Model Change, type=CONTACT PAIR, remove';

step_code_current10='_PickedSurf68,_PickedSurf64';

step_code_current $11=^{\prime}$ 'Model Change, type=CONTACT PAIR, remove';

step_code_current12='_PickedSurf69,';

step_code_current $13={ }^{\prime} *$ Restart, write, frequency $=0$;

eval(['step_code_current14="*Output, field, variable=PRESELECT, time interval=',num2str(output_interval),"'])

eval(['step_code_current15="*Output, history, variable=PRESELECT, time interval=',num2str(output_interval),"']) step_code_current $16={ }^{\prime} *$ End Step';

step_code_new $=$...

[step_code_new;step_code_current 1 ;step_code_current 2 ;step_code_current 3 ;step_code_current 4 ;step_code_current5 $; \ldots$ 
step_code_current6;step_code_current 7 ;step_code_current 8 ;step_code_current 9 ;step_code_current $10 ;. .$. step_code_current 11 ;step_code_current12;step_code_current 13 ; step_code_current14;step_code_current $15 ;. .$. step_code_current16];

else eval(['step_code_current1="*Step, name=Winding',num2str(i),', nlgeom=YES, inc=',num2str(increment),"']) step_code_current $2=$ '*Dynamic';

eval(['step_code_current3="',num2str(step_ini),,',,num2str(time_end),,',,num2str(step_min),"''])

eval(['step_code_current4="** Name: BC-',num2str(i),' Type: Displacement/Rotation"']);

step_code_current5='*Boundary';

eval(['step_code_current6="_PickedSet',num2str(1000+i),', 1, 1, ',num2str(RESULT(i,1),10),"']])

eval(['step_code_current7="_PickedSet',num2str(1000+i),', 2, 2, ',num2str(RESULT(i,2),10),"'])

step_code_current $8=' *$ Restart, write, frequency $=0$;

eval(['step_code_current9="*Output, field, variable=PRESELECT, time interval=',num2str(output_interval),"'])

eval(['step_code_current10="*Output, history, variable=PRESELECT, time interval=',num2str(output_interval),"']) step_code_current $11={ }^{\prime}$ End Step';

step_code_new=[step_code_new;step_code_current 1 ;step_code_current $2 ;$ step_code_current $3 ;$ step_code_current 4 ;ste p_code_current $5 ; \ldots$

step_code_current6;step_code_current7;step_code_current8;step_code_current9;step_code_current10;... step_code_current11;];

end

end 